\title{
Resurgence of Scrub Typhus: An Overview
}

\author{
Suvendu Jana ${ }^{1}$, Poulami Karmakar ${ }^{2}$, Partha S Karmakar ${ }^{3}$
}

\begin{abstract}
Scrub typhus is a widely neglected disease, which is gaining global momentum because of its resurgence patterns. The disease is now being reported in newer regions as well aspreviously endemic areas, being called as "tsutsugamushi triangle."Typhus is a Greek word meaning fever with stupor.

Keywords: Disseminated vasculitis, Eschar, Typhus, Trombiculid mite, Tsusugamushi triangle.

Bengal Physician Journal (2021): 10.5005/jp-journals-10070-7051
\end{abstract}

\section{INTRODUCTION}

It is a re-emerging acute infectious zoonotic disease, which can get transmitted from animals to humans or, to be more specific, a disease that usually exists in animals but can cause human infections. Scrub typhus is a very serious health problem of the Asia-Pacific region; particularly endemic in "tsutsugamushi triangle" (Fig. 1) extending from Afghanistan to China, Korea, Indonesia, and in Pacific islands including part of Australia. ${ }^{1}$ This triangle is named after the causative organism Orientia tsutsugamushi. This traditional triangle includes almost upper half of India, that is northeast and northwest parts of India.

Almost 1 billion population are at risk for scrub typhus infection and 1 million new cases occur every year. ${ }^{2}$

This traditional triangle includes almost upper half of India, that is northeast and northwest parts of India.

Scrub typhus was first described in Japan in the year 1899. ${ }^{3}$ In India, it was one of the major causes of febrile illness among the military personnel in world war II along Assam-India Myanmar (Burma) border and in 1965 Indo-Pak war. ${ }^{4}$ Now in the Indian subcontinent, scrub typhus is the most common rickettsial infection. It occurs all over India from the southern part to northern India to northeast states and central India. It occurs mainly during rainy seasons and affects all ages and both sexes with male preponderance.

\section{Nomenclature}

Scrub-the type of foliage (topography between woods and clearings) which harbors the vector.

Typhus (the Greek word) - means "fever with stupor."

\section{Causative Organism}

O. tsutsugamushi, a member of the Rickettsiaceae family, is an obligate intracellular gram-negative bacteria. It differs from other members of Rickettsia since it lacks lipopolysaccharides and peptidogly can on its cell wall and has no outer slimy layer.

The true reservoir of infection is the trombiculid mite. Chigger mites are the primary reservoir for $O$. tsutsugamushi. The microbes are transmitted transovarially in mites from adult to eggs and from the eggs to the larva or adult through transstadial transmission. Thus, they maintain infection throughout their life stages. Larval

\author{
1,3Department of General Medicine, RG Kar Medical College, \\ Rampurhat, West Bengal, India \\ ${ }^{2}$ Department of General Medicine, SDMCH, Kamarhati, West Bengal, \\ India
}

Corresponding Author: Partha S Karmakar, Department of General Medicine, RG Kar Medical College, Kolkata, West Bengal, India, Phone: +91 7679634227, e-mail: parthamed@yahoo.co.in

How to cite this article: Jana S, Karmakar P, Karmakar PS. Resurgence of Scrub Typhus: An Overview. Bengal Physician Journal 2021;8(2):45-48.

Source of support: Nil

Conflict of interest: None

stage (chigger) acts as a reservoir as well as a vector for infecting humans and rodents. Small rodents are secondary reservoirs. Humans are accidental hosts. They acquire the disease by the bite of infected chiggers. No direct person-to-person transmission occurs.

O. tsutsugamushi is the causative agent and gets transmitted through the bite of thrombiculid mites-the vector. The chigger (larval) phase is the only stage that is parasitic on animals or humans.

Chiggers normally feed on thin, wrinkled, and tender skin with the formation of eschar. Common sites are below the umbilicus, in axillae, neck region, chest, and abdomen.

\section{Pathophysiology}

Chigger inoculates $O$. tsutsugamushi pathogens into the skin of human host, where it infects dendritic cells in the dermis. Thereafter regional and later generalized lymphadenopathy occurs via lymphogenous spread. ${ }^{5}$ Subsequently organism enters into the circulation and continues to proliferate on the endothelium of the small blood vessels releasing cytokines. Cytokines damage the integrity of the endothelial cells, causing fluid leakage leading to perivascular edema, platelet aggregation followed by accumulation of lymphocyte and macrophages and thereby leading to focal occlusive endangiitis, the basic pathogenic mechanism of scrub typhus (Rickettsial vasculitis). Subsequently, disseminated vasculitis occurs that involves lungs, skin, liver, brain, heart, and kidney.

(0) The Author(s). 2021 Open Access This article is distributed under the terms of the Creative Commons Attribution 4.0 International License (https:// creativecommons.org/licenses/by-nc/4.0/), which permits unrestricted use, distribution, and non-commercial reproduction in any medium, provided you give appropriate credit to the original author(s) and the source, provide a link to the Creative Commons license, and indicate if changes were made. The Creative Commons Public Domain Dedication waiver (http://creativecommons.org/publicdomain/zero/1.0/) applies to the data made available in this article, unless otherwise stated. 


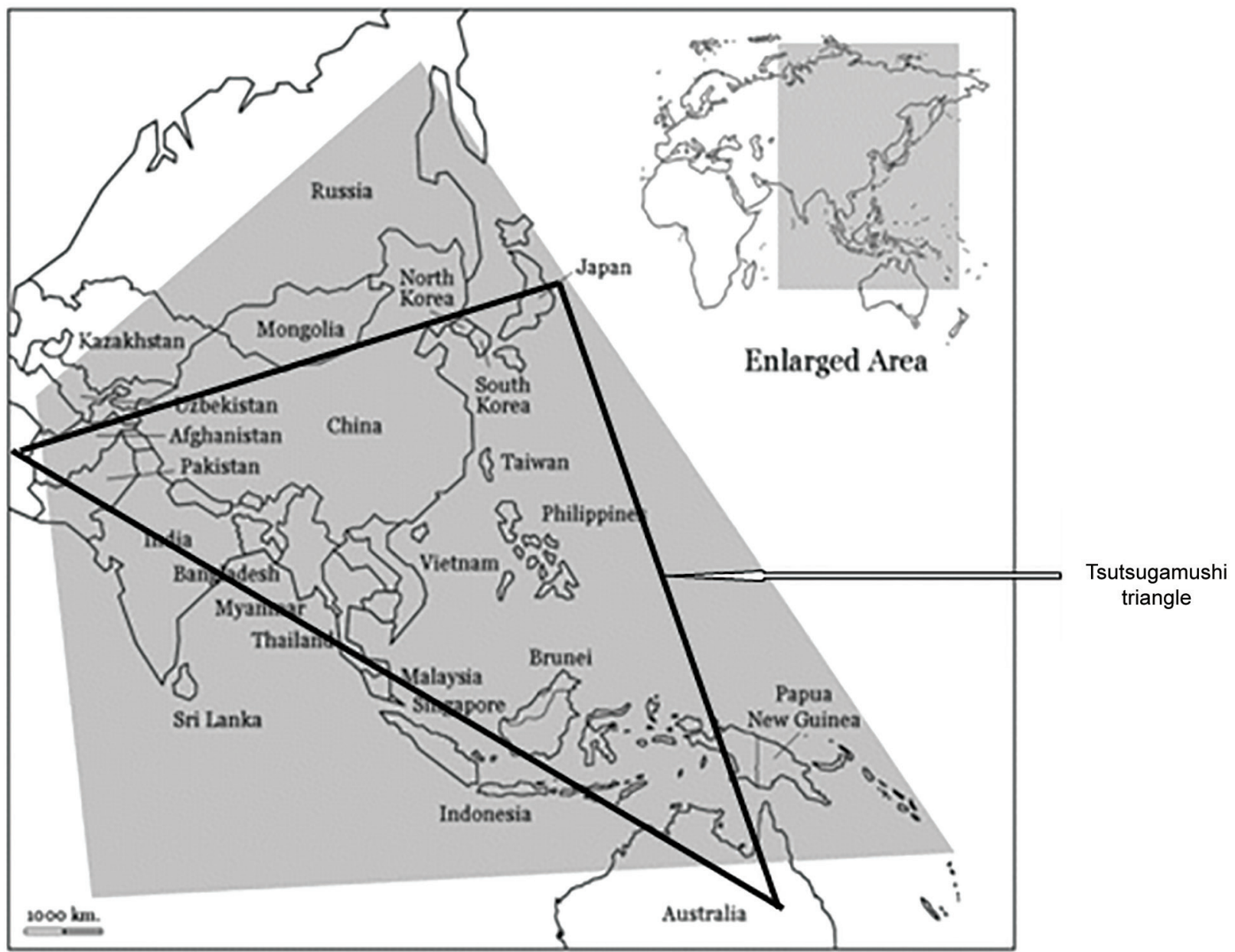

Fig. 1: Tsutsugamushi triangle (shown above): Scrub typhus is endemic in much greater area than this specified triangle

Eschar may format the inoculation site where the chigger feed. Here, it produces a painless punched-out ulcer that becomes necrotic with a black crust, surrounded by an erythematous margin.

\section{Clinical Features}

Incubation period: 6-21 days from exposure to trombiculid mite. ${ }^{1}$

Illness varies from mild to fatal disease; the majority presenting as an acute undifferentiated fever with nonspecific symptoms and signs.

\section{Nonspecific Acute Illness}

- Sudden onset fever with chill. Temperature rises from $104^{\circ} \mathrm{F}-$ $105^{\circ} \mathrm{F}$ in the first few days with relative bradycardia. Fever lasts for long period in untreated patients. Range 9-19 days (median 14.4 days).

- Headache (severe frontal headache which is more common in adults) and backache.

- Myalgia, mostly affecting lumber, thigh, and calf muscle.

- Profuse sweating.

- Conjuctival suffusion.

\section{Heightened Suspicion}

The classic case description includes eschar, rash, and lymphadenopathy.

\section{Eschar}

Painless punched-out ulcer with a black necrotic center surrounded by erythema, resembling a cigarette burn. Usually single, but multiple eschar may be found.

Size: Diameter varies from 5-20 mm.

Site: Usually found at sites of opposing skin surfaces like axilla, groin, inguinal region but may also be found in neck, chest, and abdomen.

Eschar may resolve without any sequel in 3-4 weeks.

Sometimes scarring or pigmentation may develop.

If present, it is the single most important clue for diagnosis.

\section{Rash}

Nonpruritic maculopapular rash develops in fewer than $40 \%$ of patients. It is usually seen around 5-8 days after onset of fever, typically begins in trunk and spreads to extremities. Face is also often involved. 


\section{Lymphadenopathy}

Tender lymph node, usually proximal to the site of mite bite.

\section{Complications}

In severe cases, microangiopathies involve multiple organs. Complications are usually developed after first week of sickness. It is directly related to tsutsugamushi level in the blood.

Pulmonary complications: Well documented complicationARDS. The primary pathological process of lung involvement is interstitial pneumonia associated with or without vasculitis. Oldage, thrombocytopenia, and the presence of early pneumonitis are the risk factors for the development of ARDS.

Neurological complications: Meningitis, meningoencephalitis, cranial nerve palsy.

Cardiac dysfunction: Myocarditis and cardiomegaly congestive heart failure.

Acute hepatic failure

Acute renal failure

Acute pancreatitis and peritonitis

Disseminated intravascular coagulation

Multiorgan disorder syndrome

\section{Diagnosis}

In a patient with undifferentiated fever, scrub typhus is diagnosed based on a high level of clinical suspicion.

\section{Laboratory Diagnosis}

Leukocytosis or leucopenia may be present, but mostly normal WBC count. Lymphocyte count is decreased. Thrombocytopenia may be sufficient to cause bleeding. Liver enzymes are elevated in $75-95 \%$ cases. There may be increased bilirubin, raised alkaline phosphatases, hypoalbuminemia, and raised creatinine.

The most commonly reported abnormalities in chest X-ray include bilateral, diffuse, and reticulonodular opacities. ${ }^{6,7}$ There may be minimal pleural effusion, hilar adenopathy and nonhomogenous opacities, and cardiomegaly.

\section{Serological Tests}

Weil-Felix Test: Most cost effective and most widely available serological test. It detects cross reacting antibodies to proteus mirabilis OXK. This test is of little value in the early diagnosis of scrub typhus since agglutinating antibodies IgM are detected only after 5th day of sickness and not before the appearance of the characteristic rash. The diagnostic test shows greater than four times rise in titer to OXK in paired serum taken 2 weeks apart during the febrile period. However, the test lacks sensitivity and specificity. Only less than $50 \%$ of those infected with scrub typhus have a strongly positive or rising Weil-Felix titer. Scrub typhus reinfection does not usually cause a second rise in the titer of OXK agglutinins.

Indirect Immunofluorescence Antibody: It detects specific antibody from patient's serum bound to smear of scrub typhus antigen. The results are available within 2 hours and are considered gold standard.

ELISA: Qualitative ELISA for detection of IgM antibodies is now commercially available. Primary infection leads to a rapid rise in
IgM antibody titer within 8 days, while reinfection is detected by the sharp rise of IgG titer with a variable IgM response.

In the clinical setting, if a patient with AFI presents with eschar and a reactive IgM ELISA for scrub typhus, the diagnosis is considered. Even when an eschar is absent, a diagnosis of scrub typhus has to be considered if IgM ELISA is positive and there is defervescence occurring within 48 hours of initiation of doxycycline treatment, provided other causes of AFI have been ruled out with appropriate investigation. ${ }^{8}$

Isolation of O. tsutsugamushi: O. tsutsugamushi can be isolated from the defibrinated whole blood, buffy coat of heparinized blood, plasma, triturated clot, skin biopsy, necropsy tissue, and arthropod samples. ${ }^{9}$ Embryonated eggs were previously used widely but now cell culture techniques have replaced them. Cell culture method is most widely used in order to separate Orientia from the clinical samples.

\section{TREATMENT}

Treatment should be initiated early based on a presumptive diagnosis. Rapid defervescence is highly characteristic and so it is used as a diagnostic test. Fever normallysubsides within 24-72 hours of starting the antibiotic.

Doxycycline $100 \mathrm{mg}$ twice daily orally for 7-14 days is the treatment of choice. Treatment for a shorter duration may cure the disease but it is associated with relapse.

Tetracycline $500 \mathrm{mg}$ four times daily for 7-14 days may be used with similar efficacy as doxycycline.

For pregnant women and small children, the drug of choice is azithromycin. It is also recommended in doxycycline-resistant cases. Although it is used as $500 \mathrm{mg}$ once daily for 3-5 days, the exact dose and duration are not clear as yet.

Though rifampicin can also be used as an alternative drug, it should not be used alone because of the possibility of development of resistance. It may be used in combination with doxycycline or azithromycinin places where there is an inadequate response to doxycycline alone. ${ }^{10}$ However it should be avoided in tuberculosis endemic areas like India.

\section{Prevention}

Mite control-this includes

- Mite infected areas should be avoided

- Wearing protective clothing

- Individual prophylaxis by impregnating clothes with miticidal chemicals (benzyl benzoate and permethrin) and by using mite repellents on exposed surfaces of the skin (such as diethyl toluamide)

- Application of chlorinated hydrocarbons to the vegetations and ground in selected regions (such as dieldr in, lindane, and chlordane).

Chemoprophylaxis-Doxycycline $200 \mathrm{mg}$ once weekly is found to be effective. ${ }^{10}$ It should be considered for nonimmune people and in high risk travellers.

Vaccine: There is no effective vaccine due to large amount of antigenic variation. ${ }^{10}$ 


\section{Reasons for Resurgence of Scrub Typhus}

A tremendous explosion of scrub typhus over a decade has been observed and the following factors could be attributed for this.

- Drastic reduction in the use of tetracycline and chlormycetin (both have satisfactoryanti-scrub typhus activity) over more than past two decades for various causes of febrile episode because of the availability of better antibiotics with less/no activity against scrub typhus

- Rapid deforestation over the past few decades and the use of terrain for living and cultivation has created favourable environment for the scrub grass and consequently facilitating the laying of eggs by a mite, a vector for scrub typhus. ${ }^{11}$

\section{References}

1. Sharma P, Kakkar R, Kaore SN, et al. Geographical distribution, effect of season \& life cycle of scrub typhus. JK Sci 2010;12(2):63-64.

2. Watt G, Parola P. Scrub typhus and tropical rickettsioses. Curr Opin Infect Dis 2003;16(5):429-436. DOI: 10.1097/00001432-20031000000009.

3. Kumar D, Raina DJ, Gupta S, et al. Epidemology of scrub typhus. JK Sci 2010;12(2):60-62.
4. Viswanathan $\mathrm{S}$, Muthu V, Iqbal N, et al. Scrub typhus meningitisin South India - a retrospective study. PLoS One 2013;8(6):e66595. DOI: 10.1371/journal.pone.0066595.

5. Paris DH, Phetsouvanh R, Tanganuchitcharnchai A, et al. Orientia tsutsugamushi in human scrub typhus eschars shows tropism for dendritic cells and monocytes rather than endothelium. PLoS Negl Trop Dis 2012;6(1):e1466. DOI: 10.1371/journal.pntd.0001466.

6. Choi YH, Kim SJ, Lee JY, et al. Scrub typhus: radiological and clinical findings. Clin Radiol 2000;55(2):140-144. DOI: 10.1053/crad.1999.0336.

7. Song WS, Kim KT, Ku YM, et al. Clinical role of interstitial pneumonia in patients with scrub typhus: a possible marker of disease severity. $J$ Korean Med Sci 2004;19(5):668-673. DOI: 10.3346/jkms.2004.19.5.668.

8. Dasch GA, Halle S, Bourgeois AL. Sensitive microplate enzyme linked immunosorbent assay for the detection of antibodies against scrub typhus rickettsia, Rickettsia tsutsugamushi. J Clin Microbiol1979;9(1):38-48. DOI: 10.1128/jcm.9.1.38-48.1979.

9. Chrispal A, Boorugu H, Gopinath KG, et al. Acute undifferentiated febrile illness in adult hospitalized patients: the disease spectrum and diagnostic predictors-an experience from a tertiary care hospital South India. Trop Doct 2010;40(4):230-234. DOI: 10.1258/ td.2010.100132.

10. Ramasubramanian V, Senthur Nambi P.Scrub typhus. In:Muruganathan A, Geetha T, editors. Medicine update, vol. 23.2013. p. 19-22.

11. Min KD, Lee JY,So Y, et al. Deforestation increases the risk of scrub typhus in Korea. Int J Environ Res Public Health 2019;16(9):1518. DOI: 10.3390/ijerph16091518. 